\title{
Warnhinweise in den Wind geschrieben
}

INSOMNIE

\section{Was Patienten (nicht) verstehen}

Forscher haben überprüft, ob die Warnund Gebrauchshinweise in den Beipackzetteln von Medikamenten beim Verbraucher richtig ankommen. 500 Probanden bekamen zunächst einen herkömmlichen Beipackzettel, danach einen, der in einer möglichst einfachen Sprache formuliert war. Den herkömmlichen Beipackzettel interpretierten die Versuchspersonen zu $80 \%$ richtig, den sprachlich überarbeiteten zu $91 \%$. Nun fand die Studie im englischen Sprachraum statt. Aber die Beipackzettellyrik ist sicher auch bei uns nicht jedem verständlich. Was spricht z.B. dagegen, statt „nur für den externen Gebrauch“" zu schreiben: „Darf nur auf die Haut aufgetragen werden"? Arch Intern Med.2010;170(1):50-56

\section{LEBENSSTILFAKTOREN}

\section{Das lässt die Haut alt aussehen}

Hautalterungsprozesse werden schätzungsweise zu 40\% durch den Lebensstil bestimmt. Welche Faktoren dabei eine Rolle spielen, haben Wissenschaftler bei 65 Zwillingspaaren untersucht. Lichtschäden in Form von starken Falten, Altersflecken und erweiterten Blutgefäßen fanden sich häufiger bei Studienteilnehmern, die rauchten, übergewichtig waren, auf Sonnenschutzmittel verzichteten oder früher an Hautkrebs erkrankt waren.

Arch Dermatol 2009;145:1375-79

\section{Zu wenig Schlaf, zu wenig Hirn?}

Chronische Schlafstörungen können zu einem Verlust von Hirnsubstanz führen. In einer Studie mit voxelbasierter Morphometrie fand sich bei Patienten mit chronischer Insomnie im Vergleich zu Personen ohne Schlafprobleme weniger graue Substanz im linken orbitofrontalen Kortex. Der Substanzverlust war umso ausgeprägter, je schwerer die Schlaflosigkeit von den Patienten empfunden wurde. Biological Psychiatriy, 2010;67(2):182

\section{BLUTHOCHDRUCK}

\section{Was mit Ernährung und Sport zu erreichen ist}

Bei übergewichtigen Patienten mit Hypertonie lässt sich der Blutdruck allein durch Lebensstiländerungen drastisch reduzieren. In früheren Studien wurde bereits der blutdrucksenkende Effekt der sog. DASH-Diät (wenig Fett, rotes Fleisch, Zucker, Natrium, viel Obst, Gemüse, Vollkorn, Fisch) belegt. Noch wirksamer ist ,es, wenn die Patienten zusätzlich ihr Gewicht reduzieren und Sport machen. In einer Studie mit 144 Probanden (RR 130159/85-99 mmHg) sank der Blutdruck innerhalb von vier Monaten unter der DASH-Diät um 11,2/7,5 mmHg und unter DASH-Diät plus Sport und Gewichtsabnahme um 16,1/9,9 mmHg - verglichen mit einem Rückgang um 3,4/3,8 mmHg bei unverändertem Lebensstil. Arch Intern Med 2010;170(2):126-35

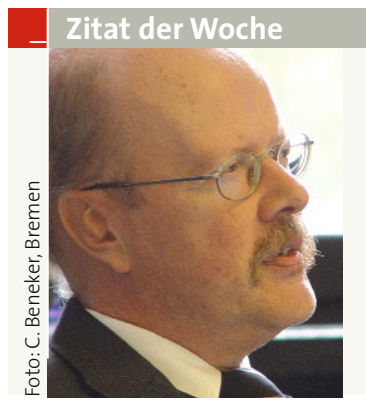

\section{"Non-Compliance ist eine Volkskrank- heit, an der 20 Millionen Bundesbürger leiden und 40000 pro Jahr sterben."}

Dr. Werner Kissling, Leiter des Centrums für Disease Management, Psychiatrische Klinik der Technischen Universität München

\section{MMW aufgefrischt}

Wir haben die MMW einer Frühjahrskur unterzogen. Das betrifft nicht nur das Layout, mit dem die MMW ab der nächsten Ausgabe noch frischer, moderner, lesbarer daherkommen wird. Sie können sich auch auf einige neue Rubriken und Leseangebote freuen, mit denen wir noch mehr auf Ihre Bedürfnisse in der Praxis eingehen möchten.

Die MMW-Telefon-Hotline für Ihre Fragen zum Praxismanagement und zur Abrechnung steht Ihnen ab nächster Woche zur Verfügung.

In unserer neuen Serie zur Naturheilkundlichen Medizin können Sie Ihr Behandlungsangebot ergänzend zur Schulmedizin erweitern.

Unsere Rubrik „Arztgeschichten“ lädt Sie zum Austausch von Erlebnissen aus dem Berufsalltag ein - Erfahrungen mit unvergesslichen Patienten, bemerkenswerten Kollegen und Mitarbeitern zum Schmunzeln, Nachdenken und Über-deneigenen-Tellerrand-Hinausschauen.

\section{All dies und mehr ab nächster Woche} in Ihrer MMW!

\section{PÄDIATRIE}

\section{Heimtückische Klobrillen}

Pädiater des Johns Hopkins Children Center warnen vor einem Phänomen, das sie in letzter Zeit bei kleinen Kindern wieder gehäuft beobachten: der Klobrillendermatitis. Klobrillen aus exotischen Hölzern, mit besonderen Lackierungen und der Gebrauch von aggressiven Desinfektionsmitteln hinterlassen Spuren auf zarten Kinderpos. Im Prinzip wäre die Dermatitis leicht zu behandeln indem man die noble Klobrille gegen ein vulgäres Plastikexemplar austauscht. Auch britische Pädiater warnen vor den schweren hölzernen Klobrillen. Sie beobachten gehäuft Fälle, in denen kleine Jungs, die ihr kleines Geschäft verrichten wollten, traumatische Erfahrungen mit bösartig zuklappenden Holzklodeckeln machten.

Pediatrics 2010; doi:10.1542/peds.2009-2430 